\title{
Qualidade de mudas de Copaifera langsdorffii Desf., cultivadas em substratos alternativos, em função de adubação complementar
}

Rubens Ribeiro da Silva, Andréia Brandão de Melo Lopes Rêgo, Bruno Aurélio Campos Aguiar, Gllson Araújo de Freitas, Pietro Lopes Rêgo, Priscila Bezerra de Souza

Universidade Federal do Tocantins - UFT. E-mail: aguiar.florestal@gmail.com

\section{Resumo}

Atualmente, é crescente o interesse na produção de mudas de espécies nativas para recuperação de áreas degradadas, recomposição de reserva legal e áreas de preservação permanente ou para o uso em diferentes setores da economia. Com isso objetivou-se avaliar a qualidade de mudas de Copaifera langsdorffii Desf. (Copaíba) cultivada em substrato alternativo em função da adubação mineral complementar. O experimento foi desenvolvido no viveiro florestal da Universidade Federal do Tocantins UFT, campus universitário de Gurupi. Instalou-se ensaio experimental em delineamento de blocos casualizados, em esquema fatorial de $2 \times 12$, com dez repetições. Foram mensurados aos 90 dias após a emergência: altura das mudas, comprimento da raiz; diâmetro do colo; massa seca de parte aérea e sistema radicular; área foliar; densidade do substrato e o índice de qualidade de mudas. $\mathrm{O}$ substrato foi constituído por húmus de minhoca (50\%) e terra de subsolo (50\%) na ausência de adubação proporciona melhoria na morfologia das mudas quanto a massa seca da parte aérea, massa seca das raízes e o maior índice de qualidade de mudas. Os substratos com $75 \%$ comercial e $25 \%$ de terra de subsolo e $100 \%$ de substrato comercial não requerem a suplementação da fertilidade com adubação para promover a melhoria no índice de qualidade de mudas. No entanto, se forem utilizadas as fontes de substrato comercial e casca de arroz carbonizada, recomenda-se o uso de adubação à medida em que forem diminuindo a proporção desses compostos orgânicos.

Palavras-chave: copaíba; desenvolvimento de mudas; índice de Dickson; morfologia.

Quality of Copaifera langsdorffii Desf., cultivated in alternative substrates, in complementary fertilization function

\begin{abstract}
There is a growing interest in the production of seedlings of native species for the recovery of degraded areas, restoration of legal reserves and permanent preservation areas or for use in different sectors of the economy. The objective of this study was to evaluate the quality of seedlings of Copaifera langsdorffii Desf. (Copaiba) cultivated in alternative substrate as a function of complementary mineral fertilization. The experiment was carried out in the forest nursery of the Federal University of Tocantins - UFT, Gurupi university campus. The experimental design was a randomized block design in a $2 \times 12$ factorial scheme with ten replications. They were measured at 90 days after emergence: seedling height, root length; neck diameter; dry mass of shoot and root system; leaf area; substrate density and seedling quality index. The substrate consisted of earthworm humus (50\%) and underground soil $(50 \%)$ in the absence of fertilization. Substrates with $75 \%$ commercial and $25 \%$ subsoil and $100 \%$ commercial substrate do not require fertilization supplementation to promote seedling quality improvement. However, if commercial substrate and carbonized rice husk sources are used, fertilizer use is recommended as the proportion of these organic compounds decreases.
\end{abstract}

Keywords: copaíba; development of seedlings; Dickson index; morphology. 


\section{Introdução}

A propagação de espécies arbóreas nativas pode apresentar peculiaridades que dificultam a adoção do conhecimento existente para as outras espécies cultivadas em larga escala. Assim, o conhecimento adquirido com as espécies exóticas, como o gênero Eucalpytus, ou nativas, como a Handroanthus impetiginosus (Mart. ex DC) Mattos) (Ipê-Roxo) e o Theobroma cacao var. leiocarpum (Bernoulli) Cif (Cacaueiro), precisa ser aplicado em testes para as demais espécies da flora brasileira (RAMOS et al., 2015; ALVES; FREIRE, 2017; IBRAHIM et al., 2019).

Entretanto, o sucesso do plantio e do estabelecimento inicial de espécies arbóreas nativas com fins econômicos ou conservacionistas depende, em grande parte, da qualidade morfofisiológica de suas mudas, que por sua vez é dependente da qualidade dos substratos usados na produção de mudas (LORENZI, 2008).

$\mathrm{Na}$ elaboração de substratos para produção de mudas com espécies nativas, têm sido usadas diferentes matérias primas, como: terra de subsolo (Latossolo Vermelho Amarelo distrófico) a casca de arroz carbonizada, serragem, turfa, vermiculita, composto orgânico, esterco bovino, moinha de carvão, bagaço de cana e areia lavada (DUTRA et al., 2015).

Os materiais orgânicos adicionados ao substrato podem disponibilizar os nutrientes em diferentes períodos, a depender da estabilidade da formação molecular da matéria prima, da composição química, da biomassa microbiana, do equilíbrio químico, da temperatura e da umidade do meio (GONDIN et al., 2015). Todos esses fatores podem definir se o efeito na disponibilidade de nutrientes será imediato ou efeito residual, devido ao processo mais lento de decomposição. Assim, a coincidência da disponibilidade de nutrientes com a necessidade das plantas é dependente de muitos fatores externos além de apenas da composição das matérias primas.

A espécie Copaifera langsdorffii possui necessidades específicas de nutrientes, exigindo o suprimento em quantidade, época de aplicação e forma adequada. No caso da formação de mudas, a principal fonte fornecedora de nutrientes é o substrato, que deve conter os nutrientes necessários para $\mathrm{o}$ pleno desenvolvimento das mudas (SANTOS et al., 2016). Entretanto, isso nem sempre é possível, havendo a necessidade de complementar o fornecimento dos nutrientes através da adubação mineral (FAQUIN, 2005).

Quando se objetiva elaborar substratos para espécies de crescimento lento, ou de baixa exigência nutricional, a compatibilidade da oferta nutricional é facilitada. Contudo, quando o objetivo é a formação de plântulas mais exigentes em nutrientes, faz-se necessária a suplementação nutricional. Diante disso o presente trabalho teve por objetivo avaliar a qualidade de mudas de Copaifera langsdorffii Desf. em função de substratos e adubação mineral complementar.

\section{Material e Métodos}

O experimento foi desenvolvido no viveiro florestal da Universidade Federal do Tocantins - UFT, campus universitário de Gurupi, localizada nas coordenadas geográficas, $11^{\circ} 43^{\prime} \mathrm{S}$ de latitude, $49^{\circ} 04^{\prime} \mathrm{O}$ de longitude e altitude de 280 metros. Segundo a classificação de Köppen, o clima da região é B1 $W A^{\prime} a^{\prime}$ úmido com moderada deficiência hídrica, definido como tropical quente e úmido com estação chuvosa no verão e seca no inverno, precipitação anual varia entre 1500 a $2100 \mathrm{~mm}$, com a temperatura média anual situando entre $25^{\circ} \mathrm{C}$ e 26 으 (SEPLAN, 2012).

$\mathrm{O}$ ensaio experimental foi instalado em delineamento de blocos casualizados, sob cobertura do tipo sombrite com $50 \%$ de retenção da luminosidade, em esquema fatorial $2 \times 12$, com 10 repetições, com 10 plântulas por parcela. 0 primeiro fator correspondente à presença e ausência de adubação suplementar, e o segundo corresponde aos 12 substratos alternativos utilizados no experimento.

Dentro do primeiro fator a presença de adubação mineral complementar foi realizada com a adição de $2 \mathrm{~kg}$ de calcário $+1,5 \mathrm{~kg}$ de superfosfato simples $+0,5 \mathrm{~kg}$ de sulfato de amônia para cada $1000 \mathrm{~L}$ de substrato, aos cinco dias antes da instalação do experimento.

Os frutos de Copaifera langsdorfii foram coletados em cinco árvores matrizes em um fragmento de cerrado sensu stricto, localizado na região sul do estado do Tocantins, em seguida foram levadas ao laboratório de sementes florestais da Universidade Federal do Tocantins, onde as sementes foram beneficiadas e selecionadas, manualmente, eliminando as que apresentavam injurias e defeitos.

As mudas foram produzidas com semeadura de três unidades por recipiente. As mesmas foram semeadas em sacos de polietileno 
(20x30 cm, sendo diâmetro de boca e altura, respectivamente), preenchidos com os substratos correspondente a cada tratamento. 0 desbaste foi efetuado quando as mudas atingiram $4 \mathrm{~cm}$ de altura, deixando apenas a mais robusta para as avaliações das variáveis biométricas.
Os componentes utilizados na elaboração dos substratos foram húmus de minhoca (HM), casca de arroz carbonizada (CAC), substrato comercial (SC) e terra de subsolo (TS) (Tabela 1).

Tabela 1. Composição dos substratos elaborados para a produção das mudas de Copaifera langsdorffii Desf., Gurupi-TO, 2016.

\begin{tabular}{ccc}
\hline HM + TS & CAC + TS & SC + TS \\
\hline $100 \%$ de HM & $100 \%$ de CAC & $100 \%$ de SC \\
$75 \%$ de HM $+25 \%$ de TS & $75 \%$ de CAC + 25\% de TS & $75 \%$ de SC + 25\% de TS \\
$50 \%$ de HM $+50 \%$ de TS & $50 \%$ de CAC $+50 \%$ de TS & $50 \%$ de SC + 50\% de TS \\
$25 \%$ de HM $+75 \%$ de TS & $25 \%$ CAC + 75\% de TS & $25 \%$ de SC + 75\% de TS \\
\hline
\end{tabular}

A terra de subsolo (TS), utilizada nas composições dos substratos foi coletada na região Sul do Estado do Tocantins em área de cerrado sensu stricto, sem histórico de cultivo agrícola ou pecuária na área. Possui composição granulométrica com 230 g. $\mathrm{kg}^{-1}$ de argila, 75 g. $\mathrm{kg}^{-1}$ de silte e 695 g. $\mathrm{kg}^{-1}$ de areia total e composição mineral (Tabela 2).

Tabela 2. Resultado da análise química da terra de subsolo utilizada na composição dos substratos, Gurupi - TO, 2016.

\begin{tabular}{ccc}
\hline Determinação & Unidade & Quantidade \\
\hline $\mathrm{pH} \mathrm{CaCl}$ & - & 4,2 \\
$\mathrm{P} \mathrm{meh}$ & $\mathrm{mg} \cdot \mathrm{dm}^{-3}$ & 1,6 \\
$\mathrm{~S}_{\mathrm{SO}}{ }^{-2}$ & $\mathrm{mg} \cdot \mathrm{dm}^{-3}$ & 4 \\
$\mathrm{~K}$ & $\mathrm{mg} \cdot \mathrm{dm}^{-3}$ & 21 \\
$\mathrm{~K}$ & $\mathrm{cmol} \cdot \mathrm{dm}^{-3}$ & 0,05 \\
$\mathrm{Ca}$ & $\mathrm{cmol}_{\mathrm{c}} \cdot \mathrm{dm}^{-3}$ & 0,5 \\
$\mathrm{Mg}$ & $\mathrm{cmol}_{\mathrm{c}} \cdot \mathrm{dm}^{-3}$ & 0,2 \\
$\mathrm{Al}$ & $\mathrm{cmol} \cdot \mathrm{dm}^{-3}$ & 0,8 \\
$\mathrm{H}+\mathrm{Al}$ & $\mathrm{cmol} \cdot \mathrm{dm}^{-3}$ & 3,10 \\
$\mathrm{M} . \mathrm{O}$. & $\mathrm{dag} \cdot \mathrm{kg}^{-1}$ & 0,7 \\
$\mathrm{C} \cdot \mathrm{O}$ & $\mathrm{dag} \cdot \mathrm{kg}^{-1}$ & 0,4 \\
$\mathrm{~B}$ & $\mathrm{mg} \cdot \mathrm{dm}^{-3}$ & 0,1 \\
$\mathrm{Cu}$ & $\mathrm{mg} \cdot \mathrm{dm}^{-3}$ & 0,7 \\
$\mathrm{Fe}$ & $\mathrm{mg} \cdot \mathrm{dm}^{-3}$ & 24 \\
$\mathrm{Mn}$ & $\mathrm{mg} \cdot \mathrm{dm}^{-3}$ & 2,8 \\
$\mathrm{Zn}$ & $\mathrm{mg} \cdot \mathrm{dm}^{-3}$ & 0,3 \\
$\mathrm{SB}$ & $\mathrm{cmol} \cdot \mathrm{dm}^{-3}$ & 0,75 \\
$\mathrm{CTC}$ & $\mathrm{cmol} \cdot \mathrm{dm}^{-3}$ & 3,85 \\
\hline
\end{tabular}

A composição físico-químicas dos componentes utilizados na elaboração dos substratos estão dispostas na Tabela 3. 
Tabela 3. Composição físico-químicas das matérias primas utilizadas na elaboração dos substratos, Gurupi TO, 2016.

\begin{tabular}{|c|c|c|c|c|c|c|c|}
\hline \multirow[b]{2}{*}{ Análises } & \multirow[b]{2}{*}{ Und. } & \multicolumn{2}{|c|}{$\begin{array}{l}\text { Casca de Arroz } \\
\text { Carbonizada }\end{array}$} & \multicolumn{2}{|c|}{ Substrato Comercial } & \multicolumn{2}{|c|}{ Húmus de Minhoca } \\
\hline & & $\begin{array}{l}\text { Base } \\
\text { Seca } \\
110 \cong C\end{array}$ & $\begin{array}{l}\text { Umidade } \\
\text { Natural }\end{array}$ & $\begin{array}{l}\text { Base } \\
\text { Seca } \\
110 \text {-C }\end{array}$ & $\begin{array}{l}\text { Umidade } \\
\text { Natural }\end{array}$ & $\begin{array}{c}\text { Base } \\
\text { Seca } \\
110^{\circ} \mathrm{C}\end{array}$ & $\begin{array}{c}\text { Umidade } \\
\text { Natural }\end{array}$ \\
\hline $\mathrm{pH} \mathrm{CaCl}{ }_{2}$ 0,01M (Ref. & $\mathrm{pH}$ & $\begin{array}{l}--- \\
\end{array}$ & 5,20 & $\begin{array}{l}---- \\
-1\end{array}$ & 5,70 & ---- & 7,30 \\
\hline $\begin{array}{l}1: 2,5) \\
\text { Densidade }\end{array}$ & $\mathrm{g} / \mathrm{cm}^{3}$ & --- & 0,11 & ---- & 0,39 & --- & 1,47 \\
\hline $\begin{array}{l}\text { Umid. Perdida a 60- } \\
65{ }^{\circ} \mathrm{C}\end{array}$ & $\%$ & ---- & 1,29 & ---- & 27,44 & ---- & 43,8 \\
\hline $\begin{array}{l}\text { Umid. Perdida entre } \\
65 \text { e } 110^{\circ} \mathrm{C}\end{array}$ & $\%$ & ---- & 2,04 & ---- & 1,15 & ---- & 37,2 \\
\hline Umidade Total & $\%$ & ---- & 3,33 & ---- & 28,59 & ---- & 58,4 \\
\hline Materiais Inertes & $\%$ & ---- & 0,00 & ---- & 0,00 & ---- & \\
\hline Nitrogênio Total & $\%$ & 0,29 & 0,28 & 0,25 & 0,18 & 1,93 & 1,90 \\
\hline $\begin{array}{l}\text { Mat. Orgânica Total } \\
\text { (Combustão) }\end{array}$ & $\%$ & 84,61 & 81,79 & 61,27 & 43,75 & 403,3 & 402,1 \\
\hline Orgânica & & & & & & & \\
\hline $\begin{array}{l}\text { Compostável } \\
\text { (Titulação) }\end{array}$ & $\%$ & 63,99 & 61,86 & 49,05 & 35,02 & 374,7 & 359,1 \\
\hline Mat. Orgânica & & & & & & & \\
\hline $\begin{array}{l}\text { Resistente a } \\
\text { Compostagem }\end{array}$ & $\%$ & 20,62 & 19,93 & 12,22 & 8,73 & 182,07 & 181,03 \\
\hline $\begin{array}{l}\text { Carbono } \quad \text { Total } \\
\text { (Orgânico e Mineral) }\end{array}$ & $\%$ & 47,01 & 45,44 & 34,04 & 24,31 & ---- & ---- \\
\hline Carbono Orgânico & $\%$ & 35,55 & 34,37 & 27,25 & 19,46 & ---- & ---- \\
\hline Resíduo Mineral Total & $\%$ & 15,71 & 15,19 & 39,18 & 27,98 & ---- & ---- \\
\hline $\begin{array}{l}\text { Resíduo } \quad \text { Mineral } \\
\text { Insolúvel }\end{array}$ & $\%$ & 14,20 & 13,73 & 0,00 & 0,00 & ---- & ---- \\
\hline Resíduo & $\%$ & 1,51 & 1,46 & 39,18 & 27,98 & ---- & ---- \\
\hline $\begin{array}{l}\text { Solúvel } \\
\text { Relação C/N (C Total e }\end{array}$ & ---- & $162 / 1$ & $162 / 1$ & $136 / 1$ & $135 / 1$ & $10 / 1$ & $10 / 1$ \\
\hline $\begin{array}{l}\text { N Total) } \\
\text { Relação } \quad \mathrm{C} / \mathrm{N} \quad \text { (C }\end{array}$ & ---- & $123 / 1$ & $123 / 1$ & $109 / 1$ & $108 / 1$ & $7 / 1$ & $7 / 1$ \\
\hline Orgânico e N Total) & & & & & & & \\
\hline Fósforo $\left(\mathrm{P}_{2} \mathrm{O}_{5}\right.$ Total) & $\%$ & 0,22 & 0,21 & 0,49 & 0,35 & 2,13 & 2,05 \\
\hline Potássio ( $\mathrm{K}_{2} \mathrm{O}$ Total) & $\%$ & 0,31 & 0,30 & 0,36 & 0,26 & 1,89 & 1,72 \\
\hline Cálcio (Ca Total) & $\%$ & 0,20 & 0,19 & 1,92 & 1,37 & 5,60 & 5,43 \\
\hline Magnésio (Mg Total) & $\%$ & 0,10 & 0,10 & 0,46 & 0,33 & 0,90 & 0,90 \\
\hline Enxofre (S Total) & $\%$ & 0,01 & 0,01 & 0,27 & 0,19 & 0,45 & 0,38 \\
\hline Boro (B Total) & $\mathrm{mg} / \mathrm{kg}$ & 3,00 & 3,00 & 8,00 & 6,00 & 2,78 & 2,78 \\
\hline Cobre (Cu Total) & $\mathrm{mg} / \mathrm{kg}$ & 4 & 4 & 7 & 5 & 2 & 2 \\
\hline Ferro (Fe Total) & $\mathrm{mg} / \mathrm{kg}$ & 804 & 777 & 3496 & 2496 & 761 & 653 \\
\hline Manganês (Mn Total) & $\mathrm{mg} / \mathrm{kg}$ & 190 & 184 & 335 & 239 & 583 & 752 \\
\hline Zinco (Zn Total) & $\mathrm{mg} / \mathrm{kg}$ & 43 & 42 & 32 & 23 & 68 & 65 \\
\hline
\end{tabular}

Para avaliação do efeito dos substratos e uso da adubação mineral complementar sobre as mudas de Copaifera langsdorffii Desf., foram mensurados o diâmetro do colo com paquímetro digital graduado em milímetro, e altura das mudas e comprimento da raiz, utilizando uma régua graduada em milímetro. 
A área foliar foi calculada por meio de imagem usando o software ImageJ, (http://rsbweb.nih.gov/ij/). As folhas foram retiradas das mudas e identificado ao qual tratamento foram submetidas, colocando-as sobre uma folha de papel de tamanho $21 \times 29,7$ $\mathrm{cm}$ (A4), contendo impresso um retângulo de cor preta, totalmente preenchido, medindo $3 \times 15 \mathrm{~cm}$ $\left(45 \mathrm{~cm}^{2}\right)$ utilizado para calibração do software. Em seguida, foram fotografadas todas as folhas das mudas de Copaifera langsdorffii Desf. com auxílio de uma máquina digital com 12.0 megapixels de resolução, e posteriormente foram submetidas à análise de área através de algoritmos de contrastes e modelos matemáticos do software ImageJ versão 1.48.

Foram determinadas aos 90 dias pósemergência, a massa seca da parte aérea e da raiz das mudas, para tanto, elas foram previamente preparadas, sendo cuidadosamente destorroadas e a parte aérea então separada do sistema radicular. A qualidade das mudas segundo Dickson et al. (1960) foi mensurada pela equação 1 .

$$
I Q D=\frac{M S T p}{\frac{A M}{D C}+\frac{M S A}{M S R}} \quad \text { Equação (1) }
$$

Em que: MSTp = peso da matéria seca total $(\mathrm{g}), \mathrm{AM}=$ altura das mudas $(\mathrm{cm}), \mathrm{DC}=$ diâmetro do colo $(\mathrm{mm}), \mathrm{MSA}=$ peso da matéria seca da parte aérea $(\mathrm{g})$ e MSR = peso da matéria seca da raiz (g). A pesagem foi realizada após a completa secagem das mudas em estufa de passagem de ar forçada a $60^{\circ} \mathrm{C}$ durante 72 horas.

Os resultados obtidos foram submetidos ao teste de normalidade (Shapiro-Wilk) e homogeneidade (Barlett) e quando significativos foi relizada à análise de variância e as médias comparadas através do teste de Tukey ao nível de probabilidade de $5 \%$ com uso do software Sisvar (FERREIRA, 2011).

\section{Resultados e Discussão}

$\mathrm{Na}$ análise de variância não foi encontrado efeito significativo do fator adubação mineral complementar para os valores dos parâmetros diâmetro do colo, massa seca da raiz, índice de qualidade de mudas e densidade do substrato. Entretanto, para as demais variáveis analisadas, altura das mudas, área foliar, comprimento radicular e massa seca da parte aérea, o resultado foi significativo (Tabela 4).

Tabela 4. Resumo do quadro de análise de variância para altura de mudas (AM), área foliar (AF), diâmetro do colo (DC), comprimento radicular (CR), massa seca da parte aérea (MSPA), massa seca da raiz (MSR), índice de qualidade de Dickson (IQD) e densidade do substrato (DS. Sub), em função da fonte de variação (Adubação/Substrato), Gurupi - TO, 2016.

\begin{tabular}{|c|c|c|c|c|c|c|c|c|}
\hline \multirow[t]{2}{*}{ FV } & $\begin{array}{l}\text { AM } \\
(\mathrm{cm})\end{array}$ & $\begin{array}{c}\mathrm{AF} \\
\left(\mathrm{cm}^{2}\right)\end{array}$ & $\begin{array}{c}\mathrm{DC} \\
(\mathrm{mm})\end{array}$ & $\begin{array}{c}\text { CR } \\
(\mathrm{cm})\end{array}$ & $\begin{array}{c}\text { MSPA } \\
\text { (g) }\end{array}$ & $\begin{array}{c}\text { MSR } \\
\text { (g) }\end{array}$ & IQD & $\begin{array}{l}\text { Ds. Sub. } \\
\left(\mathrm{kg} / \mathrm{m}^{3}\right)\end{array}$ \\
\hline & \multicolumn{8}{|c|}{ QM } \\
\hline Subs. (F1) & $105,43^{* *}$ & $2000,46^{* *}$ & $1,29 * *$ & $143,45^{* *}$ & $5,87 * *$ & $3,24 * *$ & $0,20 * *$ & $0,40^{* *}$ \\
\hline Adub. (F2) & $75,94^{* *}$ & $1524,16^{* *}$ & $0,27 \mathrm{~ns}$ & $253,13^{* *}$ & $4,91 * *$ & $0,01 \mathrm{~ns}$ & $0,01 \mathrm{~ns}$ & $0,00 \mathrm{~ns}$ \\
\hline Int. F1 x F2 & $16,67 *$ & $1115,55^{* *}$ & $0,53 * *$ & $71,68 * *$ & $4,95 * *$ & $2,10 * *$ & $0,20 * *$ & $0,01 * *$ \\
\hline CV(\%) & 17,30 & 0,25 & 11,76 & 0,78 & 2,83 & 3,19 & 12,32 & 6,05 \\
\hline
\end{tabular}

FV: Fonte de variação ${ }^{*}$ significativo ao nível de $1 \%$ de probabilidade; ${ }^{*}$ significativo ao nível de $5 \%$ de probabilidade; ${ }^{\text {ns }}$ não significativo), pelo teste de F.

As mudas de Copaifera langsdorffii Desf. que apresentaram maiores alturas, foram produzidas nos substratos $100 \%$ de $\mathrm{HM}$ e $75 \%$ de $\mathrm{HM}+25 \%$ TS sem adubação mineral complementar e $50 \%$ de $\mathrm{HM}+50 \%$ de TS com adubação suplementar, isso pode estar relacionado à boa fertilidade e o alto teor de nitrogênio presente nessa composição orgânica (Tabela 5).

O húmus de minhoca (HM), segundo Armond (2016) possui matéria orgânica, nutrientes minerais prontamente assimiláveis pelas raízes e microrganismos, favorecendo a decomposição da matéria orgânica e aumento da disponibilidade de nutrientes oriundos da ciclagem de nutrientes, tais características são importante e contribuem para o maior crescimento das mudas.

A casca de arroz carbonizada (CAC) usada na composição do substrato apresentou as mudas de menores alturas devido a sua grande porosidade, isso pode ter contribuído para a perda de nutrientes e atrelado à baixa contribuição nutricional. Resultados parecidos foram encontrado por Araújo e Paiva Sobrinho (2011), que analisaram a germinação e produção 
de mudas de tamboril (Enterolobium Contortisiliquum (Vell.) Morong) em diferentes substratos e por Paiva Sobrinho et al. (2010) que analisaram o crescimento de mudas de mangabeira (Hancornia speciosa Gomes), cumburareiro (Dipteryx alata Vog.) e cagaiteira (Eugenia dysenterica DC).

A área foliar das mudas de Copaifera langsdorffii Desf. apresentou diferenças estatísticas em resposta as combinações de substrato com e sem a adição da adubação suplementar. Dentre os tratamentos que receberam adubação suplementar, o melhor desenvolvimento da área foliar foi verificado nas combinações em $25 \%$ de $\mathrm{HM}+75 \%$ de TS e $100 \%$ de HM (Tabela 5).

Tabela 5. Altura de mudas e área foliar de mudas de Copaifera langsdorffii, produzidas em substratos adubados (ad) e não adubados (n-ad), Gurupi-TO, 2016.

\begin{tabular}{|c|c|c|c|c|}
\hline \multirow{2}{*}{ Substratos } & \multicolumn{2}{|c|}{ Altura de Mudas $(\mathrm{cm})$} & \multicolumn{2}{|c|}{ Área Foliar $\left(\mathrm{cm}^{2}\right)$} \\
\hline & ad & n-ad & ad & n-ad \\
\hline $25 \%$ de SC $+75 \%$ de TS & 14,35 bcB & $18,45 \mathrm{abA}$ & $70,50 \mathrm{fA}$ & $62,93 \mathrm{gB}$ \\
\hline $50 \%$ de $\mathrm{SC}+50 \%$ de TS & 15,25 bcA & 15,85 bcdA & $78,70 \mathrm{cdA}$ & $44,73 \mathrm{iB}$ \\
\hline $75 \%$ de SC $+25 \%$ de TS & $14,90 \mathrm{bcB}$ & $18,00 \mathrm{abcA}$ & $78,20 \mathrm{~dB}$ & $111,05 \mathrm{cA}$ \\
\hline $100 \%$ de SC & $18,45 \mathrm{abA}$ & $18,55 \mathrm{abA}$ & $78,72 \mathrm{cdB}$ & $116,34 \mathrm{bA}$ \\
\hline $25 \%$ de $\mathrm{HM}+75 \%$ de TS & 15,85 bcB & $18,45 \mathrm{abA}$ & $106,84 \mathrm{aA}$ & $99,76 \mathrm{~dB}$ \\
\hline $50 \%$ de $\mathrm{HM}+\mathbf{5 0} \%$ de TS & 20,20 aA & $19,60 \mathrm{abA}$ & $52,26 \mathrm{hB}$ & $111,09 \mathrm{cA}$ \\
\hline $75 \%$ de $\mathrm{HM}+25 \%$ de TS & $17,80 \mathrm{abcB}$ & $20,40 \mathrm{aA}$ & 51,67 iB & $70,57 \mathrm{fA}$ \\
\hline $100 \%$ de HM & $17,55 \mathrm{abcB}$ & 20,65 aA & $97,61 \mathrm{bB}$ & 124,35 aA \\
\hline $25 \%$ CAC $+75 \%$ de TS & $14,05 \mathrm{cA}$ & $13,55 \mathrm{dA}$ & $79,21 \mathrm{cA}$ & 72,71 eB \\
\hline $50 \%$ de CAC $+50 \%$ de TS & 14,25 bcA & $13,60 \mathrm{dA}$ & $75,38 \mathrm{eA}$ & $63,38 \mathrm{gB}$ \\
\hline $75 \%$ de CAC $+25 \%$ de TS & $13,95 \mathrm{cA}$ & $13,95 \mathrm{cdA}$ & $69,86 \mathrm{gA}$ & $52,21 \mathrm{hB}$ \\
\hline $100 \%$ de CAC & 14,30 bcA & $13,35 \mathrm{dA}$ & $52,57 \mathrm{hB}$ & $72,82 \mathrm{eA}$ \\
\hline
\end{tabular}

*Médias seguidas de mesma letra não diferem estatisticamente entre si, maiúscula na linha e minúscula na coluna, pelo teste de Tukey a $5 \%$ de probabilidade. $\mathrm{SC}=$ substrato comercial; $\mathrm{HM}=$ Húmus de minhoca; $\mathrm{CAC}=$ Casca de arroz carbonizada e TS $=$ terra de subsolo.

As mudas que apresentaram maior diâmetro do colo foram às cultivadas nos substratos em composição do húmus de minhoca, sendo as combinações de $100 \%$ de HM com adubação mineral complementar e $75 \%$ de
HM sem adubação mineral complementar que promoveram os maiores incrementos em diâmetro do colo (Tabela 6). 
Tabela 6. Diâmetro do colo e comprimento radicular de mudas de Copaifera langsdorffii, produzidas em substratos adubados (ad) e não adubados (n-ad), Gurupi-TO, 2016.

\begin{tabular}{|c|c|c|c|c|}
\hline \multirow{2}{*}{ Substratos } & \multicolumn{2}{|c|}{ Diâmetro do Colo (mm) } & \multicolumn{2}{|c|}{ Comprimento Radicular (cm) } \\
\hline & ad & n-ad & ad & n-ad \\
\hline $25 \%$ de SC $+75 \%$ de TS & $2,50 \mathrm{bcA}$ & $2,10 \mathrm{deB}$ & $34,33 \mathrm{bB}$ & $41,33 \mathrm{aA}$ \\
\hline $50 \%$ de SC $+50 \%$ de TS & $2,40 \mathrm{bcdA}$ & 2,40 bcdeA & $27,00 \mathrm{fB}$ & $41,00 \mathrm{aA}$ \\
\hline $75 \%$ de SC $+25 \%$ de TS & $2,65 \mathrm{abA}$ & $2,65 a b A$ & $24,66 \mathrm{gB}$ & $28,00 \mathrm{fA}$ \\
\hline $100 \%$ de SC & $2,70 \mathrm{abA}$ & $2,70 \mathrm{abA}$ & $25,33 \mathrm{gB}$ & 32,66 eA \\
\hline $25 \%$ de $H M+75 \%$ de TS & $2,45 \mathrm{bcA}$ & $2,45 \mathrm{bcdA}$ & $32,83 \mathrm{cB}$ & $38,66 \mathrm{cA}$ \\
\hline $50 \%$ de $H M+50 \%$ de TS & $2,00 \mathrm{~dB}$ & $2,70 \mathrm{abA}$ & $34,00 \mathrm{bA}$ & $23,66 \mathrm{hB}$ \\
\hline $75 \%$ de $H M+25 \%$ de TS & $2,60 \mathrm{abcB}$ & $3,00 \mathrm{aA}$ & $31,33 \mathrm{dA}$ & $27,66 \mathrm{fB}$ \\
\hline $100 \%$ de HM & $3,00 \mathrm{aA}$ & $2,60 \mathrm{abcB}$ & $19,66 \mathrm{hB}$ & $25,33 \mathrm{gA}$ \\
\hline $25 \%$ CAC $+75 \%$ de TS & $2,20 \mathrm{cdA}$ & 2,20 cdeA & $24,66 \mathrm{gB}$ & $33,66 \mathrm{dA}$ \\
\hline $50 \%$ de CAC $+50 \%$ de TS & $2,00 \mathrm{~dB}$ & $2,50 \mathrm{bcdA}$ & $29,66 \mathrm{eB}$ & 39,83 bA \\
\hline $75 \%$ de CAC $+25 \%$ de TS & $2,30 \mathrm{bcdA}$ & 2,30 bcdeA & 38,66 aA & $39,00 \mathrm{cA}$ \\
\hline $100 \%$ de CAC & $2,00 \mathrm{dA}$ & $2,00 \mathrm{eA}$ & $31,33 \mathrm{dA}$ & $27,66 \mathrm{fB}$ \\
\hline
\end{tabular}

*Médias seguidas de mesma letra não diferem estatisticamente entre si, maiúscula na linha e minúscula na coluna, pelo teste de Tukey a $5 \%$ de probabilidade. SC = substrato comercial; $\mathrm{HM}=$ Húmus de minhoca; $\mathrm{CAC}=\mathrm{Casca}$ de arroz carbonizada e TS = terra de subsolo.

Os menores valores de massa seca da parte aérea com utilização da adubação mineral complementar foram observados nos substratos $25 \%$ de SC $+75 \%$ de TS, $25 \%$ de HM $+75 \%$ de TS, $25 \%$ CAC $+75 \%$ de TS e $100 \%$ de CAC. As mudas produzidas nas diferentes proporções de casca de arroz carbonizadas e na composição de $25 \%$ de
SC $+75 \%$ de TS sem o uso da adubação suplementar, apresentaram menores massa seca da parte área, quando comparado aos demais substratos sem adubação mineral complementar (Tabela 7).

Tabela 7. Massa seca da parte aérea e da raiz de mudas de Copaifera langsdorffii Desf., produzidas em substratos adubados (ad) e não adubados (n-ad), Gurupi-TO, 2016.

\begin{tabular}{|c|c|c|c|c|}
\hline \multirow{2}{*}{ Substratos } & \multicolumn{2}{|c|}{ Massa Seca da Parte Aérea (g) } & \multicolumn{2}{|c|}{ Massa Seca da Raiz (g) } \\
\hline & ad & n-ad & ad & n-ad \\
\hline $25 \%$ de $S C+75 \%$ de TS & $1,88 \mathrm{eB}$ & $2,19 \mathrm{gA}$ & $2,20 \mathrm{dA}$ & $2,15 \mathrm{gA}$ \\
\hline $50 \%$ de $\mathrm{SC}+\mathbf{5 0} \%$ de TS & $3,80 \mathrm{aA}$ & $1,71 \mathrm{hB}$ & $2,67 \mathrm{cA}$ & 2,71 efA \\
\hline $75 \%$ de $S C+25 \%$ de TS & $2,81 \mathrm{~dB}$ & $4,87 \mathrm{cA}$ & $3,36 \mathrm{bA}$ & $3,45 \mathrm{dA}$ \\
\hline $100 \%$ de SC & $3,28 \mathrm{bB}$ & $3,87 \mathrm{dA}$ & $3,27 \mathrm{bA}$ & $3,24 \mathrm{dA}$ \\
\hline $25 \%$ de $\mathrm{HM}+75 \%$ de TS & $1,95 \mathrm{eB}$ & $5,19 \mathrm{bA}$ & $2,40 \mathrm{~dB}$ & $4,47 \mathrm{bA}$ \\
\hline $50 \%$ de $\mathrm{HM}+\mathbf{5 0 \%}$ de TS & $2,90 \mathrm{~dB}$ & $6,42 \mathrm{aA}$ & $3,89 a B$ & $4,84 \mathrm{aA}$ \\
\hline $75 \%$ de $\mathrm{HM}+25 \%$ de TS & $4,04 \mathrm{aB}$ & $5,31 \mathrm{bA}$ & $3,43 \mathrm{bB}$ & $3,91 \mathrm{cA}$ \\
\hline $100 \%$ de HM & $4,03 \mathrm{aA}$ & $3,57 \mathrm{eB}$ & $4,07 \mathrm{aA}$ & $2,53 \mathrm{fB}$ \\
\hline $25 \%$ CAC $+75 \%$ de TS & $1,84 \mathrm{eB}$ & $2,41 \mathrm{fgA}$ & $1,53 \mathrm{eB}$ & $2,92 \mathrm{eA}$ \\
\hline $50 \%$ de $\mathrm{CAC}+50 \%$ de TS & 3,16 bcA & $1,61 \mathrm{hB}$ & $1,79 \mathrm{eB}$ & $1,99 \mathrm{gA}$ \\
\hline $75 \%$ de CAC $+25 \%$ de TS & $2,98 \mathrm{cdA}$ & $1,30 \mathrm{iB}$ & $3,18 \mathrm{bA}$ & $1,72 \mathrm{hB}$ \\
\hline $100 \%$ de CAC & $1,99 \mathrm{eB}$ & $2,48 \mathrm{fA}$ & $3,44 \mathrm{bA}$ & $1,58 \mathrm{hB}$ \\
\hline
\end{tabular}

*Médias seguidas de mesma letra não diferem estatisticamente entre si, maiúscula na linha e minúscula na coluna, pelo teste de Tukey a 5\% de probabilidade. SC = substrato comercial; $\mathrm{HM}=$ Húmus de minhoca; $\mathrm{CAC}=\mathrm{Casca}$ de arroz carbonizada e TS = terra de subsolo. 
Com a diminuição da proporção do húmus de minhoca foi observada uma elevada redução de massa seca da parte aérea para os tratamentos com uso da adubação suplementar. Isso se deve provavelmente, pela redução da disponibilidade de nutrientes quando a proporção do composto foi diminuída.

A massa seca da parte aérea de mudas de Copaifera langsdorffii Desf. que foram produzidas na composição de $75 \%$ de CAC $+25 \%$ de TS sem adubação mineral complementar apresentaram os menores valores e diferiram dos demais tratamentos. A maior produção de massa seca da parte aérea foi obtida em resposta ao cultivo no substrato em composição de $50 \%$ de $\mathrm{HM}+50 \%$ de TS sem o uso da adubação suplementar. Neste sentido, a massa seca da parte aérea foi maior nas mudas cultivadas em iguais proporções de húmus de minhoca (HM) e terra de subsolo (TS), pois observou-se decréscimos nos valores dessa variável à medida que se aumentou a proporção desse composto sem o uso da adubação suplementar.

O aumento da massa seca radicular é importante por ser um indicador de vigor e da velocidade de estabelecimento de mudas, pois uma maior área de raiz (comprimento + peso) possibilita maior acesso aos nutrientes e à água disponível no solo. Taiz e Zeiger (2009) relatam que esta variável expressa balanço funcional entre a taxa fotossintética e a absorção de água pelas raízes, que em condições normais, apresenta certo equilíbrio. Mattei (1999) acrescenta que o comprimento do sistema radicular é fundamental para o estabelecimento de uma espécie florestal em determinado ambiente.

Os maiores valores de densidade são observados quando se utiliza a menor proporção dos compostos orgânicos em composição de SC, HM e CAC independente do uso de adubação mineral complementar (Tabela 8).

Tabela 8. Médias do índice de qualidade de Dickson e da densidade do substrato de mudas de Copaifera langsdorffii Desf., produzidas em substratos adubados (ad) e não adubados (n-ad), Gurupi-TO, 2016.

\begin{tabular}{|c|c|c|c|c|}
\hline \multirow{2}{*}{ Substratos } & \multicolumn{2}{|c|}{$\begin{array}{l}\text { Densidade do Substrato } \\
\left(\mathrm{kg} \cdot \mathrm{m}^{-3}\right)\end{array}$} & \multicolumn{2}{|c|}{ Índice de Qualidade de Dickson } \\
\hline & ad & n-ad & ad & n-ad \\
\hline $25 \%$ de $S C+75 \%$ de TS & $1,12 \mathrm{aA}$ & $0,98 \mathrm{aB}$ & $0,62 \mathrm{cdA}$ & $0,44 \mathrm{fB}$ \\
\hline $50 \%$ de SC $+50 \%$ de TS & $0,87 \mathrm{bA}$ & $0,85 \mathrm{bcA}$ & $0,84 \mathrm{bcA}$ & 0,61 defB \\
\hline $75 \%$ de $S C+25 \%$ de TS & $0,62 \mathrm{cA}$ & $0,64 \mathrm{dA}$ & $0,95 \mathrm{abA}$ & $1,02 \mathrm{bcA}$ \\
\hline $100 \%$ de SC & $0,41 \mathrm{eA}$ & $0,44 \mathrm{eA}$ & $0,83 \mathrm{bcA}$ & $0,88 \mathrm{bcdA}$ \\
\hline $25 \%$ de $\mathrm{HM}+75 \%$ de TS & $1,14 \mathrm{aA}$ & $1,02 \mathrm{aB}$ & $0,60 \mathrm{cdB}$ & $1,12 \mathrm{abA}$ \\
\hline $50 \%$ de $H M+50 \%$ de TS & $0,78 \mathrm{bA}$ & $0,82 \mathrm{cA}$ & $0,62 \mathrm{cdB}$ & $1,33 \mathrm{aA}$ \\
\hline $75 \%$ de $\mathrm{HM}+25 \%$ de TS & $0,54 \mathrm{cdA}$ & $0,59 \mathrm{dA}$ & $0,96 a b B$ & $1,13 \mathrm{abA}$ \\
\hline $100 \%$ de HM & $0,41 \mathrm{deB}$ & $0,57 \mathrm{dA}$ & $1,19 \mathrm{aA}$ & 0,65 defB \\
\hline $25 \%$ CAC $+75 \%$ de TS & $1,13 \mathrm{aA}$ & $0,95 a b B$ & $0,44 \mathrm{~dB}$ & 0,76 cdeA \\
\hline $50 \%$ de CAC $+50 \%$ de TS & $0,79 \mathrm{bA}$ & $0,85 \mathrm{bcA}$ & $0,56 \mathrm{dA}$ & 0,58 efA \\
\hline $75 \%$ de CAC $+25 \%$ de TS & $0,63 \mathrm{cA}$ & $0,59 \mathrm{dA}$ & 0,86 bcA & $0,44 \mathrm{fB}$ \\
\hline $100 \%$ de CAC & $0,35 \mathrm{eA}$ & $0,25 \mathrm{fB}$ & $0,70 \mathrm{bcdA}$ & $0,49 \mathrm{fB}$ \\
\hline
\end{tabular}

* Médias seguidas de mesma letra não diferem estatisticamente entre si, maiúscula na linha e minúscula na coluna, pelo teste de Tukey a $5 \%$ de probabilidade. SC = substrato comercial; HM = Húmus de minhoca; CAC = Casca de arroz carbonizada e TS = terra de subsolo.

Isso pode estar relacionado ao fato de o material orgânico apresentar baixa densidade e o aumento da sua concentração infere diretamente na redução da densidade do substrato como um todo. Na proporção de $25 \%$ dos componentes orgânicos a adubação promoveu um aumento da densidade quando comparada a mesma proporção sem adubação suplementar.
$O$ índice de qualidade de Dickson (IQD) variou entre 0,44 a 1,33 para os diferentes tratamentos, segundo Hunt (1990), valores maiores que 0,20 são considerados satisfatórios, ou seja, todos os tratamentos foram capazes de produzir mudas de qualidade. Fonseca et al., (2002), afirma que o índice Dickson é um bom determinador da qualidade de mudas, pois 
utiliza-se de dados sobre a robustez e a distribuição da biomassa.

\section{Conclusões}

Os substratos com $100 \%$ de composição comercial e $100 \%$ de húmus de minhoca proporcionam os maiores incrementos em altura e área foliar das mudas de Copaifera langsdorffii Desf.

A redução nas proporções das matérias primas promovem redução nos índices de qualidade de mudas requerendo a adubação mineral complementar para as proporções inferiores a $50 \%$, sendo essa demanda maior quando usada à casca de arroz carbonizada.

\section{Referências}

ALVES, F. J. B.; FREIRE, A. L. O. Crescimento inicial e qualidade de mudas de ipê-roxo (Handroanthus impetiginosus (Mart. Ex dc) Mattos) produzidas em diferentes substratos. Agropecuária Científica no Semiárido, v.13, n.3, p. 195-202, 2017.

ARAÚJO, A. P. de; PAIVA SOBRINHO, $S$ de. Germinação e produção de mudas de tamboril (Enterolobium contortisiliquum (Vell.) Morong) em diferentes substratos. Revista Árvore, v.35, n.3, p. 581-588, 2011.

https://doi.org/10.1590/S0100-

$\underline{67622011000400001}$

ARMOND, C.; OLIVEIRA, V. C. de; GONZALES, S. D. P.; OLIVEIRA, F. É. R. de; da SILVA, R. M.; LEAL, T. T.; REIS, A. S.; SILVA, F. da. Desenvolvimento inicial de plantas de abobrinha italiana cultivada com húmus de minhoca. Horticultura Brasileira, v.34, n.3, p. 439-442, 2016. https://doi.org/10.1590/S0102-05362016003022

DICKSON, A.; LEAF, A. L.; HOSNER, J. F. Quality appraisal of white spruce and white pine seedling stock in nurseries. Forest Chronicle, v.36, n.2, p. 10-13, 1960. https://doi.org/10.5558/tfc36010-1

DUTRA, T. R.; GRAZZIOTTI, P. H.; SANTANA, R. C.; MASSAD, M. D. Qualidade de mudas de copaíba produzidas em diferentes substratos e níveis de sombreamento. Floresta, v.45, n.3, p. 635-644, 2015. https://doi.org/10.5380/rf.v45i3.35686

FAQUIN, V. Nutrição mineral de plantas. 2. ed. Lavras: UFLA/FAEPE, 2005. 183 p.
FERREIRA, D. F. Sisvar: a computer statistical analysis system. Ciência e Agrotecnologia. v.35, n.6, p. 1039-1042, 2011.

https://doi.org/10.1590/S1413-

$\underline{70542011000600001}$

FONSECA, É. P.; VALÉRI, S. V.; MIGLIORANZA, É.; FONSECA, N. A. N.; COUTO, L. Padrão de qualidade de mudas de Trema micrantha (L.) Blume, produzidas sob diferentes períodos de sombreamento. Revista Árvore, v.26, n.4, p. 515523, 2002. https://doi.org/10.1590/S0100$\underline{67622002000400015}$

GONDIN, J. C.; SILVA, J. B. da; ALVES, C. Z.; DUTRA, A. S.; ELIAS JUNIOR, L. Emergência de plântulas de Schizolobium amazonicum Huber ex Ducke (CAESALPINACEAE) em diferentes substratos e sombreamento. Revista Ciência Agronômica, v.46, n.2, p. 329-338, 2015. https://doi.org/10.5935/1806-6690.20150012

HUNT, G. A. Effect of stryrblock design and cooper treatment on morphology of conifer seedlings. In: TARGET SEEDLINGS SYMPOSIUM MEETING OF THE WESTERN FOREST NURSERY ASSOCIATIONS, 1990, Oregon. Anais [...]. Fort Collins: United States Department of Agriculture, Forest Service, 1990. p. 218-222.

IBRAHIM, J. F. de O. N.; SILVA JUNIOR, I. V. da; COSTA BARROS, F. da; PAEZ, D. R. M.; NASCENTES, A. L.; SILVA, L. D. B. da. Utilização do lodo de esgoto na produção de mudas e no cultivo do eucalipto (Eucalyptus spp). Brazilian Journal of Animal and Environmental Research, v.2, n.1, p. 564-579, 2019.

LORENZI, H. Árvores brasileiras: manual de identificação e cultivo de plantas arbóreas nativas do Brasil. 5. ed. Nova Odessa: Instituto Plantarum, 2008. 420 p. v. 1.

MATTEI, V. L. Deformações radiculares em plantas de Pinus taeda L. produzidas em tubetes quando comparadas com plantas originadas por semeadura direta. Ciência Florestal, v.4, n.1, p. 19, 1999. https://doi.org/10.5902/19805098295

RAMOS, A.; SODRÉ, G. A.; PORTO, J. S.; QUEIROZ COSTA, R. de. Desenvolvimento de mudas de cacaueiro em função de níveis de aplicação de água. Agrotrópica, v.27, n.1, p. 19-24, 2015. 
https://doi.org/10.21757/0103-

$\underline{3816.2015 v 27 n 1 p 19-24}$

SANTOS, J. E. G.; SILVA, M. A. P. da; SILVA, D. L. da. Estrutura, dispersão e distribuição espacial de Copaifera langsdorfii DESF. na floresta nacional do Araripe, Ceará, Brasil. Cadernos de Cultura e Ciência, v.15, n.1, p. 72-81, 2016.

https://doi.org/10.14295/cad.cult.cienc.v15i1.11 $\underline{36}$

TOCANTINS (Estado). Secretaria da Fazenda e Planejamento (SEPLAN). Diretoria de Zoneamento Ecológico- Econômico (DZE). Base de dados geográfico do TO: versão 2012. Palmas, TO: SEPLAN, 2012. Disponível em: http://seplan.to.gov.br/. Acesso em: 13 jan. 2019.

PAIVA SOBRINHO, S.; LUZ, P. B. D.; SILVEIRA, T. L.; RAMOS, D. T.; NEVES, L. G.; BARELLI, M. A. Substratos na produção de mudas de três espécies arbóreas do cerrado. Revista Brasileira de Ciências Agrárias, v.5, n.2, p. 238-243, 2010. https://doi.org/10.5039/agraria.v5i2a741

TAIZ, L.; ZEIGER, E. Fisiologia vegetal. 4. ed. Porto Alegre: Artmed, 2009. 819 p. 